\title{
Shoe Size Recommendation System Based on Shoe Inner Dimension Measurement
}

\author{
Damir OMRČEN, Aleš JURCA \\ UCS, Universal Customization System, Slovenia
}

\begin{abstract}
The purpose of this work is to develop a system for supporting Internet footwear sales. The goal is to increase the accuracy of the shoe size and model selection when buying in the Internet. We propose a recommendation system which improves the classification accuracy and is based on foot and/or shoe scanners.

271 adult subjects participated in this study. The subjects were asked to estimate the comfort and fit of the shoes. The analysis show that only $50 \%$ of selections were correct if the selection relies on subject's usual size. One of the main reasons for the poor results of shoe selection is the inconsistency of shoe production. Therefore, we developed special devices for measuring the actual inner 3D model of shoes. The shoe inner dimension measurement (SID) is based on x-ray images of the shoes. Based on shoe and foot dimension we are able to increase the recommendation accuracy from $50 \%$ up to $85 \%$ in some cases. Such a progress could make the Internet shoe sales problematic feasible.
\end{abstract}

Keywords: shoe inner dimension measurement, shoe size recommendation system, fit of footwear, Internet shoe sales

\section{Introduction}

Internet is a multi-billion dollar economy and the Internet's economic potential is growing every year (BBC news, 2010). On the other hand the Internet sales in footwear lag behind. In our opinion the main reason for the poorly developed Internet footwear sales is the inability to estimate the fit of shoes.

There have been a number of attempts to make the footwear Internet sales more efficient and successful. In some cases a customer is encouraged to buy more shoe sizes, selects one and returns the others. Sometimes a special electronic or mechanical device is used to measure the feet (Boër, 2007). A good shoe size recommendation system should consider the following components in the recommendation:

- geometrical shape of the foot,

- personal preferences of the customer,

- inner dimensions of the shoe,

- technical characteristics (material, construction, ...) of the shoe.

Based on the findings we developed UCS recommendation system. One of the crucial components in our recommendation system is a foot scanner. There are number of foot scanners available on the market (Boër, 2007). Some of them use set of cameras to define the exact 3D shape of the foot. The others only produce scan of the sole. In this work we shortly describe a foot scanner, which generates a 2.5 dimensional scan in only a fraction of a second and can be successfully used in a recommendation system.

Even more significant component of a good recommendation system is measurement of the inner shape of a shoe. The shoe inner dimension is defined by the shoe last on which the shoe is produced. The shoe last, the most important component of a successful shoe manufacture, has always been the subject of a big interest. Though, a good shoe fitting depends on the finished shoe and not on the last. Namely, the inner dimensions of a shoe might considerably differ from those of the last. There were some minor attempts of measurement of the inner dimensions of shoes. In addition to casting and other destructive methods (which are not useful for a massive implementation) there were some high-tech approaches: CT and optical methods for the inner measurement (Coudert, 2007 and Richter, 2010). Both have significant drawbacks which pushed us to develop a new method (Omrcen, 2010) which is nondestructive, precise enough, fast and economically acceptable also for a massive implementation. In the following we shortly present the basic elements of our development: Non-invasive X-ray based determination of the inner dimensions of a shoe.

The main contribution of this study is in development of a recommendation system for supporting Internet footwear sales. The goal is to increase the accuracy of the shoe size and model selection when buying in the Internet. We propose a recommendation system which improves the recommendation accuracy and is based on foot and/or shoe scanners. 


\section{Method}

Initially, 271 adult subjects (107 male, 164 female) were invited to participate in the study. The subjects were asked to estimate the comfort and fit of 22 different shoe models (from different categories of footwear - from fashion to casual and sport). The subjects were asked to put the shoes on, make a few steps and then to estimate the comfort and fit of the shoes. Then for each model subjects were asked to select the one size that fits best (best size).

On the other hand the term usual size stays for the shoe size that a subject usually wears. This is also the size selected when buying in the Internet or selected as the first to try when buying in a shop.

In this study we start with the analysis of people's habits and feelings regarding the shoe fit. We compare the best shoe sizes to the usual sizes of the subjects. We evaluated the accuracy of such a selection for buying in the Internet. Since the correctness of such a selection is rather low we introduce the UCS recommendation system.

The UCS recommendation system is based on complex data mining approach. It relies on foot and inner shoe dimensions and past experiences of the customers. By introducing a complex data mining based recommendation system the recommendation accuracy increases. Using all the available information, we recommend the best shoe size for each customer and for each model. It is very important to note that the best shoe size can differ for different models. The recommendation depends on the actual shape of the shoe.

The analysis has been taken in EU sizing system (without the loss of generality).

\subsection{Feet measurement scanner}

Subject's usual size is based on past experiences of many different kinds of shoes; however, sometimes the past experiences are not good enough. Often sport shoes are smaller than declared, different sizing systems are involved in the decision, feet of young population is growing and changing, etc. Therefore, customers are not very reliable in expressing their usual size. The actual foot size sometimes differs significantly from the usual size.

To increase the reliability of the foot size data we introduce the foot measurement scanner (see Figure 1). By scanning a feet we are able to define the foot size more precisely and even to get more information about the foot scanned. The foot scanner is a fast, reliable and simple and creates 2.5D scan with the relevant measures of the foot. The scanner is described in more details in (Kolsek, 2010).

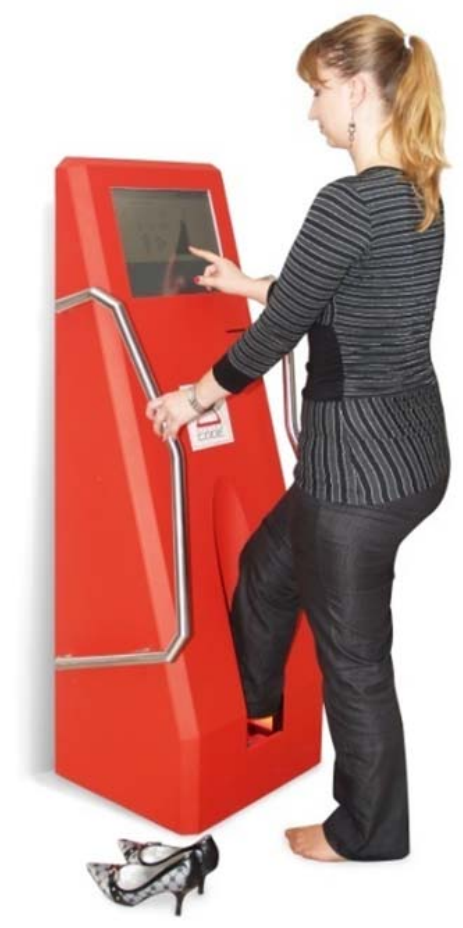

Figure 1: UCS foot scanner 
By using the relevant foot measures we define the actual foot size. The actual foot size is defined by the following equation:

$$
\text { foot size }=f(\text { length, meta. } \text { girth, instep girth, heel girth, toe shape })
$$

The foot size is a decimal number. The detailed equation is rather complex and is not given in the paper.

\subsection{Shoe inner dimension measurement}

Some shoes are smaller that declared, some are larger. Some are narrow, some are wide. Shoes differ in heel and sole silhouette. Toebox is very much affected by fashion. Therefore, to capture the complete inner volume of a shoe we developed shoe inner dimension system (SID). By using SID we are able to define the actual 3D shape of the shoe inner and to simplify, we are able to define the actual shoe size.

To measure the shoe inner dimensions and to generate the inner 3D shape of a shoe we use x-ray images of the shoe. Since the inner shape is of interest the shoe is filled with a contrast media, which increases the contrast between the shoe and the interior. By that the inner part can be automatically detected.

Currently, we use two orthogonal x-ray images to build 3D model. We use top and side image of the shoe as shown in Figure 2. Due to the high contrast it is rather easy to extract the inner part of the shoe.

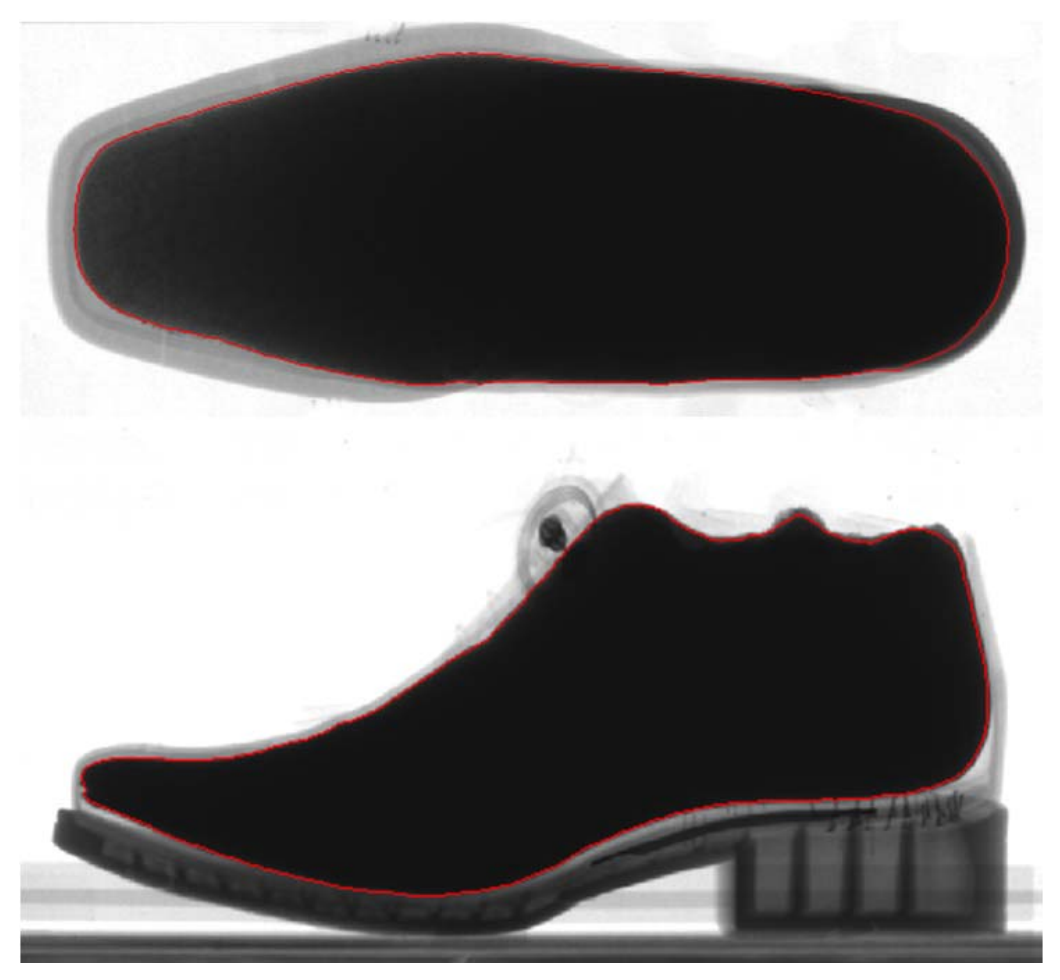

Figure 2: Top and side $x$-ray images of a shoe filled with a contrast media

Based on the two x-ray images and some additional procedures we are able to build a complete 3D model of shoe inner. The final full 3D model of the shoe is calculated and is shown in Figure 3. 


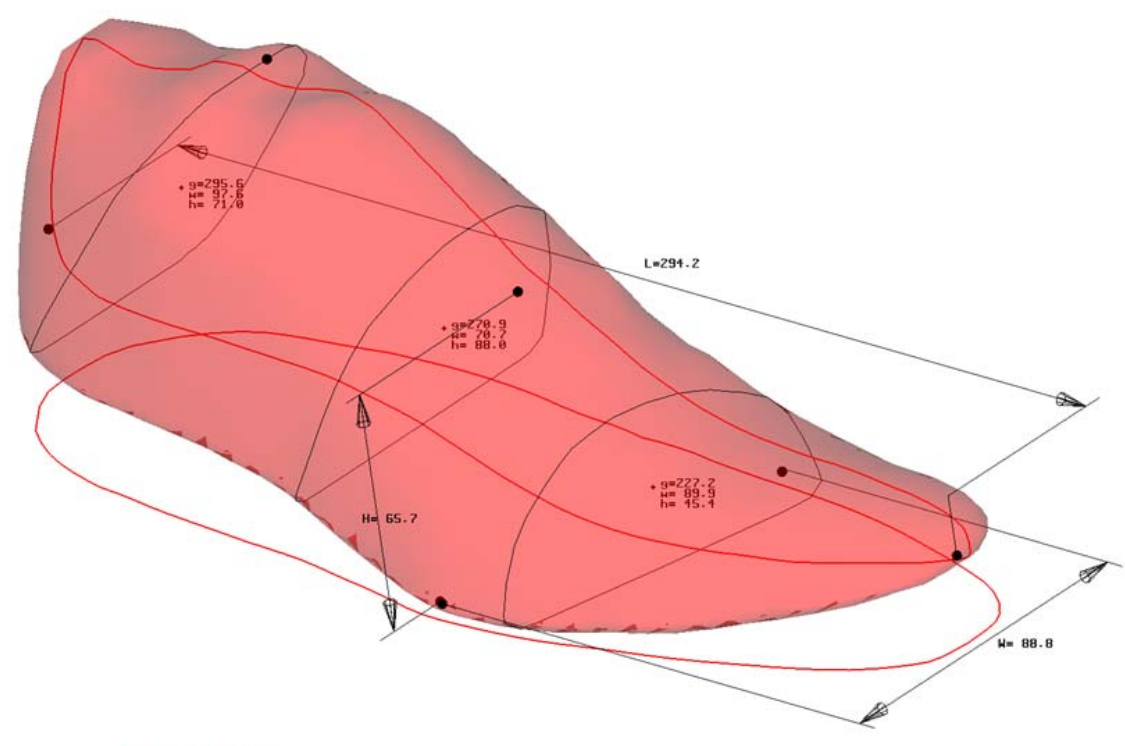

Figure 3: Full 3D model of shoe inner

The shoe measurement system is described in more details in (Omrcen, 2010). The shoe measurement process is reliable and robust and is already installed in warehouse environment. We do mass measurement of shoes and in collaboration with our partners we measure up to 30.000 pairs of shoes per year.

Based on the 3D inner shape of the shoe we can define the actual shoe size. The actual shoe size depends not only on the $3 \mathrm{D}$ shape but only on the purpose of the shoe and materials used. For example, a fashion shoe can have smaller volume comparing to a sport shoe, even though both are of the same size.

The equation for defining the actual shoe size is:

$$
\text { shoe size }=f(3 D \text { inner volume, shoe type, material }) .
$$

The shoe size is a decimal number. The detailed equation is rather complex and is not given in the paper.

Shoe inner dimension measurement is crucial for a good shoe recommendation. Additionally, one can find many useful applications for its use. For example: quality control of shoe production, analysis of shoe samples, fit improvement etc.

\subsection{Recommendation system}

In the current implementation UCS recommendation system recommends one best shoe size for each model and for each subject. First the actual foot size is defined using the foot scan. Similarly, the actual shoe size is defined using the shoe scan. Based on both information the recommendation system recommends the shoe size closest to the foot size.

The survey performed has been used in a combination with a complex data mining approach. The data mining approach has been designed in a way to maximize the recommendation accuracy. In the current implementation the goal of the data mining has been to define the formulas for defining the foot size and shoe size from their scans (see equations (1) and (2)). Using different combination of foot and/or shoe measures we search for the formulas that results in best recommendation accuracy.

Some people have very specific personal preferences. Some like more comfort shoes while the other like more tight shoes. It is impossible to detect the personal preference from a foot scan. However, it can be detected from one or more past fit estimations (or shoe purchases). We know what would be the optimal shoe size for a person and we know what the person's choice of size was. From this information we can conclude what the person's personal preference is. This preference can later be used in the recommendation system. 
Currently we are working on much more complex recommendation system. We do not only consider foot and shoe size as the only parameter. We consider the whole shape of shoe and foot to recommend the proper size with a much higher accuracy. The current results are very promising.

\section{Results}

We have compared the shoe size selected by subjects as best fit to the size normally selected when buying in the Internet (the usual size). For example:

\begin{tabular}{|l|l|c|c|c|}
\hline $\begin{array}{l}\text { Subject's } \\
\text { sizing code SC }\end{array}$ & Model name & Subject's usual size & $\begin{array}{l}\text { Best size (after } \\
\text { putting shoes on) }\end{array}$ & $\begin{array}{l}\text { Difference in size } \\
\text { (best-usual) }\end{array}$ \\
\hline 111454567897 & Claire-A & 37 & 38 & 1 \\
\hline 111454567897 & Juliet-B & 37 & 38 & 0 \\
\hline 222444455566 & Claire-A & 39 & 39 & 1 \\
\hline 222444455566 & Juliet-B & 39 & 38 & 0 \\
\hline
\end{tabular}

From this table we could conclude that person 111454567897 actual size is 38 and not 37 as expressed by the person. However, when observing all the data the conclusions are not so strait forward.

Figure 4 shows this comparison for all test shoe models. The green dotted line shows the ideal case where usual size is equal to the best size. The solid blue line shows the mean of all best sizes for a group of the same usual size subjects. As we can see the mean line is very close to the ideal line. The average distance is only 0.2 of shoe size. That means that, in average, subjects know their correct (actual) size very well.

Unfortunately, the best sizes are spread out over a large range of values. That means that many times best sizes differ from usual size significantly. Such cases result in wrong selection of the shoe size. Standard deviation (marked with $\longmapsto$ ) is approx. 0.7 of shoe size and sometimes the best size is 2 or even 3 sizes greater/smaller than the usual size (marked with $\longmapsto$ ). For example, there were subjects that have reported their usual size to be 42 but selected 45 as the best fitting size for one of the shoe model. This spread makes the Internet shoe sales very problematic.

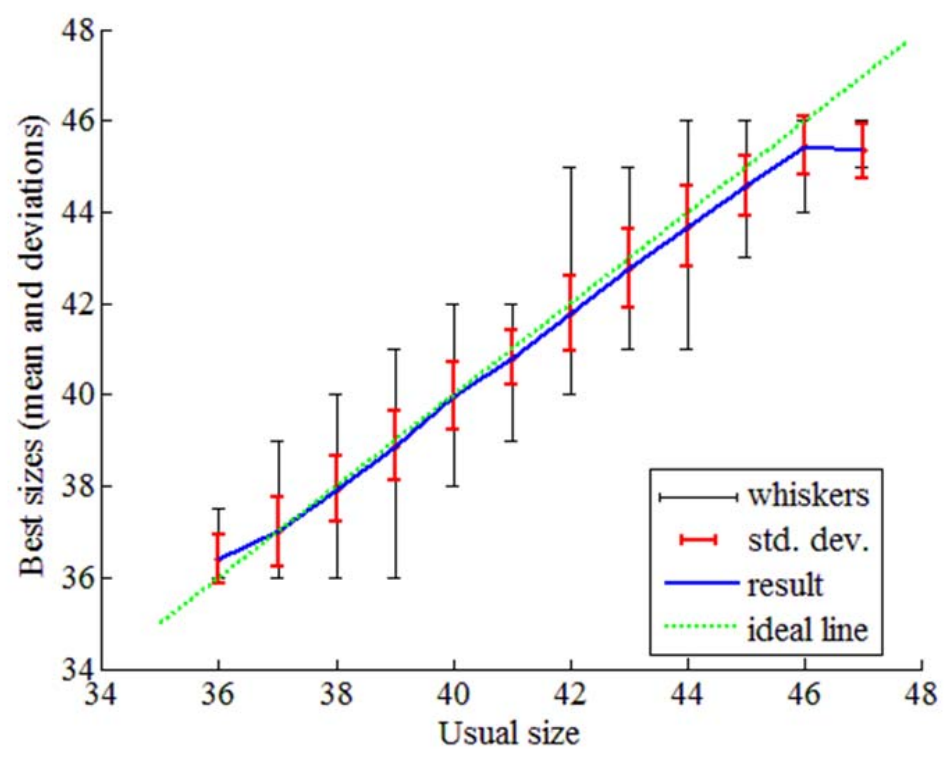

Figure 4: Comparison of best and usual size

Results have also shown that in almost $50 \%$ of cases one can fail to select the right shoe size if such selection relies only on usual size. In our opinion one of the main reasons for such a poor result in selecting the proper shoe size is the inconsistency in shoe production and poorly defined shoe size standard.

Using the methods given in section 2.3 we were able to increase the recommendation accuracy. The table below shows the recommendation accuracy using different methods: 
Table 1. Recommendation accuracy of different methods

\begin{tabular}{|l|c|}
\hline Description of the method & $\begin{array}{l}\text { Recommendation } \\
\text { accuracy (\%) }\end{array}$ \\
\hline $\begin{array}{l}\text { Normal approach (usual size defines } \\
\text { selected size) }\end{array}$ & 50 \\
\hline Use of foot and shoe scanners & 65 \\
\hline $\begin{array}{l}\text { Use foot and shoe scanners } \\
\text { Additionally considering subject's personal } \\
\text { preferences }\end{array}$ & 85 \\
\hline
\end{tabular}

Based on accurate shoe dimensions, and backed with foot dimensions, we are able, in some cases, to increase the accuracy of recommendation system from $50 \%$ up to $85 \%$.

\section{Conclusions}

We have demonstrated the innovative shoe size recommendation system. System is based on accurate foot and shoe measurements. Using those data we were able to significantly increase recommendation accuracy when selecting shoe size of the best fit.

The main goal of our work is to support Internet footwear sale. Using methods and devices proposed we believe that the Internet shoe sale could evolve.

Our future work is mainly focused in further development of the recommendation system. The goal is to increase the recommendation accuracy and to make the system less demanding. For example, we are working on a compact system that does not need the foot scanners, since those are the most problematic component for an Internet reseller. "Self foot measurement" realized at home would be a great solution for this case.

\section{References}

1. BBC News, 2011: “UK internet economy 'worth billions'”, (accessed 2011): http://www.bbc.co.uk/news/technology-11635318.

2. Boër, C.R., Dulio, S., (2007): "Mass Customization and Footwear: Myth, Salvation or Reality?", UK, Springer

3. Coudert T.,(2007): "Measurement of the inside volume of a shoe to improve comfort", In Footwear Congress

4. Richter M., (2010): "Entwicklung eines Messverfahrens zur Produktionskontrolle von Schuhinnenmaßen mit Hilfe der Computertomographie“, Project report (accessed Aug. 2011): http://pfi-germany.de/fileadmin/entwicklung eines messverfahrens.pdf

5. Omrcen, D., Kopac, M., (2010): "X-Ray Based System for Shoe Inner Dimensions Measurement", In Proc. MAT-ECO-SHOES 2010, Krakow, Poland

6. Kolsek, T., Jurca, A., Vidic, T. (2010): “Recommendation System For Sizing of Children's Footwear", In Proc. 3D Body Scanning Techniques, Switzerland 